\title{
Role of Transient Receptor Potential A1 in Gastric Nociception
}

\author{
Takashi Kondo $^{a}$ Tadayuki Oshima $^{a}$ Koichi Obata ${ }^{c}$ Jun Sakurai ${ }^{a}$ \\ Charles H. Knowles ${ }^{d}$ Takayuki Matsumoto $^{b}$ Koichi Noguchic ${ }^{c}$ Hiroto Miwa ${ }^{a}$ \\ Divisions of a Upper Gastroenterology and b ${ }^{b}$ Lower Gastroenterology, Department of Internal Medicine, \\ and ' Department of Anatomy and Neuroscience, Hyogo College of Medicine, Hyogo, Japan; ${ }^{d}$ Centre for \\ Gastroenterology, Blizard Institute of Cell and Molecular Science, Barts \& The London School of Medicine \\ and Dentistry, Queen Mary, University of London, London, UK
}

\section{Key Words \\ Visceral pain - Transient receptor potential A1 • Extracellular signal-regulated kinase $1 / 2 \cdot$ Gastric distention $\cdot$ Dorsal root ganglion $\cdot$ Nodose ganglion}

\begin{abstract}
Afferent fibers innervating the gastrointestinal tract have major roles in consciously evoked sensations including pain. However, little is known about the molecules involved in mechanonociception from the upper gastrointestinal tract. We recently reported that activation of extracellular signalregulated kinase $1 / 2$ (ERK1/2), a member of the mitogen-activated protein kinase cascade in primary afferent neurons, was induced by noxious gastric distention in the rat, and that the activation of ERK1/2 in dorsal root ganglion (DRG) neurons can be implicated in acute visceral pain. Transient receptor potential (TRP) A1, a member of the TRP family of cation channels, was expressed in both DRG and nodose ganglion (NG) neurons innervating the stomach and in nerve fibers in the gastric wall. TRPA1 was coexpressed with ERK1/2 in gastric primary afferent neurons, and attenuation of TRPA1 activation using antisense peptides and a specific blocker led to suppression of both ERK1/2 activation and visceromotor responses. TRPA1 also significantly colocalized with sub-
\end{abstract}

stance $\mathrm{P}(\mathrm{SP})$ and calcitonin gene-related peptide (CGRP) in the thoracolumbar DRG, NG and stomach. These data indicate that SP and CGRP may also be released by TRPA1 activation in primary afferent neurons to elicit neurogenic inflammation and promote visceral hyperalgesia.

Copyright $\odot 2010$ S. Karger AG, Basel

\section{Introduction}

A large group of patients with unexplained gastrointestinal problems have chronic symptoms that can be attributed to the gastroduodenal region. Of several Rome classified disorders, functional dyspepsia (FD) is numerically the most common and can be defined as the presence of one or more dyspeptic symptoms without structural or biochemical explanation [1]. Symptoms in such patients include epigastric pain, and burning, early satiation, postprandial fullness, upper abdominal bloating, excessive belching, postprandial nausea, and vomiting.

Vagal and spinal extrinsic neurons both contribute to the afferent innervation of the stomach with general recognition that the spinal component has a greater role in mediating noxious stimuli [2]. Changes in the properties of visceral sensory neurons are likely to play an important

\section{KARGER}

Fax +4161306 1234

E-Mail karger@karger.ch

www.karger.com (c) 2010 S. Karger AG, Basel

0012-2823/10/0823-0150\$26.00/0

Accessible online at:

www.karger.com/dig
Hiroto Miwa, MD, PhD

Division of Upper Gastroenterology, Department of Internal Medicine

Hyogo College of Medicine, 1-1 Mukogawa-cho

Nishinomiya, Hyogo 663-8501 (Japan)

Tel. +81 79845 6662, Fax +81 79845 6661, E-Mail miwahgi@ hyo-med.ac.jp 


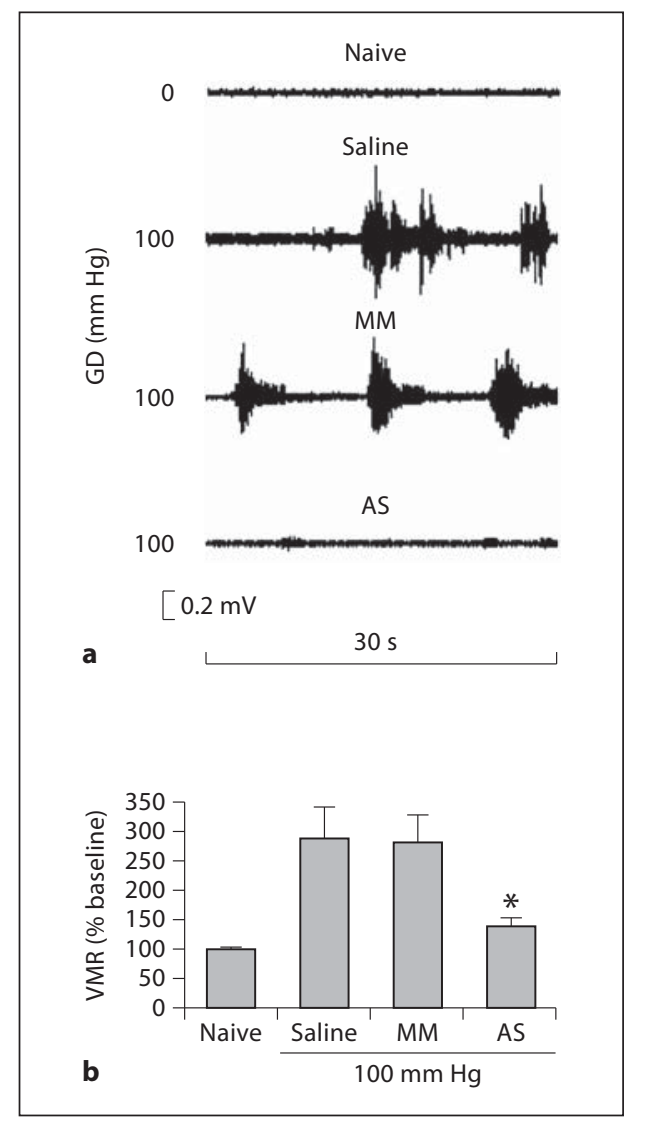

Fig. 1. VMR to GD. a Representative tracings show EMG activity recorded from the acromiotrapezius muscle 5 days after balloon implantation. b A summary of intensity-dependent changes in VMR after noxious mechanical stimulation of the stomach. 100 $\mathrm{mm} \mathrm{Hg}$ of GD caused a significant aversive response, which was prevented by TRPA1 AS-ODN (AS). Data represent mean \pm SEM; $\mathrm{n}=8$ for each group, ${ }^{*} \mathrm{p}<0.05$ vs. MM-ODN group. AS = Antisense; EMG = electromyographic; GD = gastric distention; $\mathrm{MM}=$ mismatch; $\mathrm{ODN}=$ oligodeoxynucleotide; $\mathrm{TRP}=$ transient receptor potential.

role in the development of functional gastrointestinal disorders, in particular in relation to the development of gastrointestinal pain [3]. Although it is widely acknowledged that patients with FD have evidence of visceral hypersensitivity in the stomach $[4,5]$, the cellular and molecular etiology of such hypersensitivity is relatively unstudied. Recent attention has thus started to focus on the molecules responsible for normal and heightened mechanosensation in the upper gastrointestinal tract and also on the respective contributions of spinal and vagal afferents. Here, we review the recent studies on the molecular mechanisms of gastric nociception and the mechanisms of neurogenic inflammation.

Role of Transient Receptor Potential A1 in Gastric Nociception

\section{Visceromotor Response to Gastric Distention}

Visceromotor response (VMR) to gastric distention (GD) from the acromiotrapezius muscle under slight anesthesia is a reproducible and quantifiable physiological measure used in rodents to estimate afferent responses to acute gastric nociception [6, 7]. We induced acute GD in male Sprague-Dawley rats weighing 190-260 g [5, 6], confirming that noxious levels of GD $(60,80$, and $100 \mathrm{~mm}$ $\mathrm{Hg}$ ) caused a significant and dose-dependent increase in electromyographic (EMG) activity [8] (fig. 1a).

\section{Contribution to Acute Visceral Pain of ERK1/2}

Activation in Primary Afferents

Several studies have reported that extracellular signal-regulated protein kinase 1/2 (ERK1/2) phosphorylation in spinal dorsal horn neurons and dorsal root ganglion (DRG) neurons is caused by acute noxious stimuli, such as formalin or capsaicin [9-11]. ERK1/2 is activated by an upstream kinase, mitogen-activated protein kinase (MAPK)/ERK kinase (MEK), and known to be one of the intracellular signaling pathways involved in neuronal plasticity [12]. However, the contribution to acute visceral pain of ERK1/2 activation in primary afferents was still unclear. Interestingly we found that painful stimulation of the stomach by GD induced an intensity-dependent ERK1/2 phosphorylation in both DRG and nodose ganglion (NG) neurons [8]. Small and medium-sized phosphorylated ERK1/2 (p-ERK1/2)-labeled neurons were found in DRG produced by $100 \mathrm{~mm} \mathrm{Hg} \mathrm{GD} \mathrm{(fig.} \mathrm{2a).}$ Furthermore, activation correlated well with VMR to GD [8]. These data suggest that examination of p-ERK1/2 is a very useful indicator of activated DRG neurons after noxious mechanical stimulation of the stomach in vivo.

\section{Effect of the MEK1/2 Inhibitor, U0126 on VMR to \\ Noxious GD}

To further explore the functional consequences of ERK1/2 activation in DRG neurons, we investigated whether inhibition of ERK1/2 activation modifies the response to noxious mechanical stimulation. Intrathecal (T9/10) administration of a MEK1/2 inhibitor, U0126 $(1 \mu \mathrm{g} / \mu \mathrm{l})$, significantly reduced the response to noxious mechanical stimulation of the stomach [8], and significantly suppressed the increase in the number of $\mathrm{p}$ ERK1/2-IR (immunoreactive) cells in the DRG neurons and superficial dorsal horn after GD [8]. Therefore, ERK1/2 activation in DRG neurons and/or spinal cord may itself have an important role in the pathogenesis of acute visceral pain.

Digestion 2010;82:150-155 
Fig. 2. ERK1/2 activation in TRPA1-containing neurons by noxious mechanical stimulation of the stomach. a p-ERK1/2 labeling in the T9/10 DRG neurons $2 \mathrm{~min}$ after GD of $100 \mathrm{~mm} \mathrm{Hg}$. Arrows indicate single-labeled p-ERK1/2-IR neurons. b Quantification of the percentage of $\mathrm{p}$ ERK1/2-IR neurons in the T9/10 DRG after GD of $100 \mathrm{~mm} \mathrm{Hg.} 100 \mathrm{~mm} \mathrm{Hg}$ of GD caused p-ERK1/2-IR and TRPA1 AS-ODN (AS) reversed the GD-induced p-ERK1/2IR. c Double immunostaining of TRPA1 (red) and p-ERK1/2 (green) showed that almost all of the p-ERK $1 / 2$ were colocalized with TRPAl in the DRG neurons 2 min after noxious mechanical stimulation. Data represent mean \pm SEM; $n=4$ for each group. ${ }^{*} \mathrm{p}<0.05$ vs. MM-ODN group. Scale bars: $50 \mu \mathrm{m}$. d Schema of GDinduced TRPA 1 and ERK1/2 activation in DRG and release of SP and CGRP from the peripheral terminal. AS = Antisense; CGRP = calcitonin gene-related peptide; $\mathrm{DRG}=$ dorsal root ganglion; ERK = extracellular signal-regulated protein kinase; $\mathrm{GD}=$ gastric distention; IR = immunoreactive; $\mathrm{MM}=$ mismatch ODN = oligodeoxynucleotide; $\mathrm{SP}=$ substance $\mathrm{P}$; TRP = transient receptor potential.
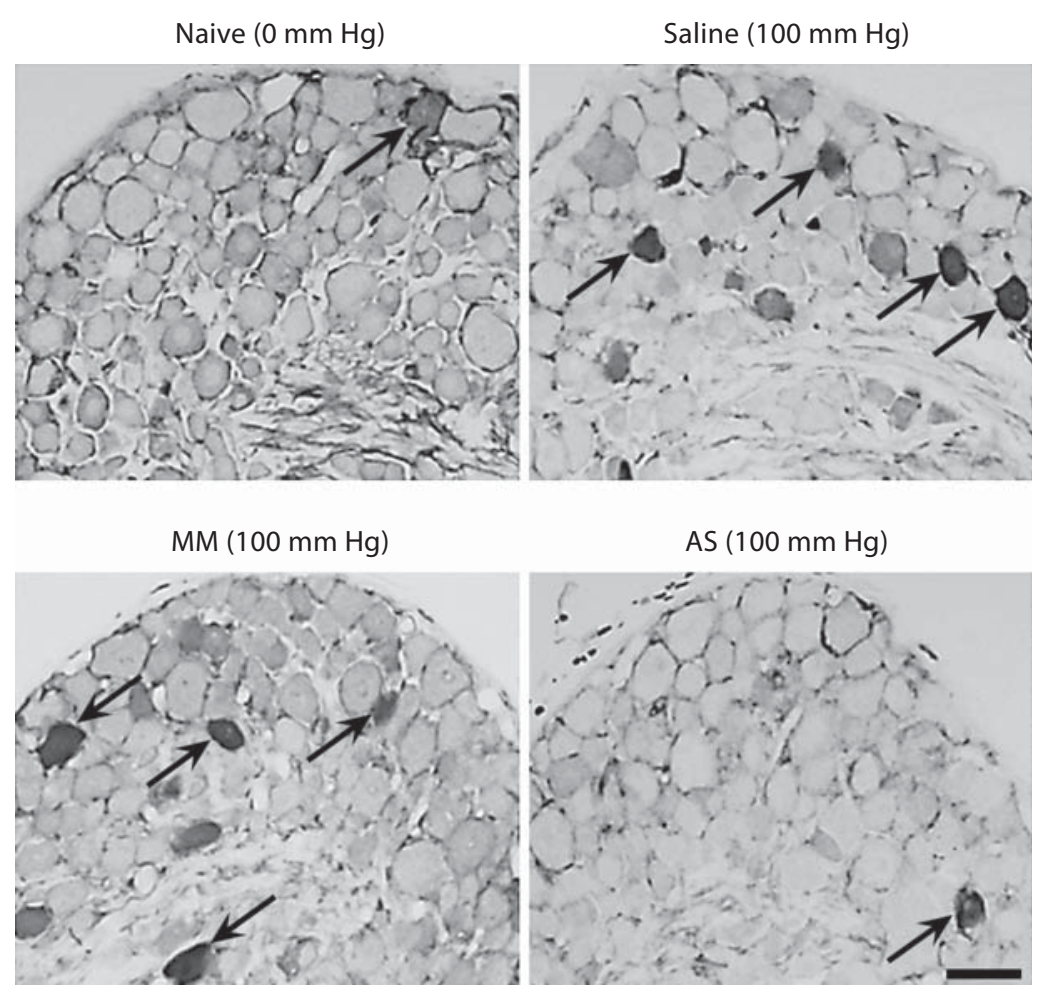

a

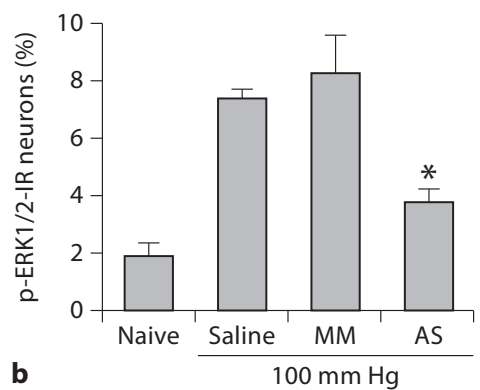

TRPA $1 / p-E R K 1 / 2$

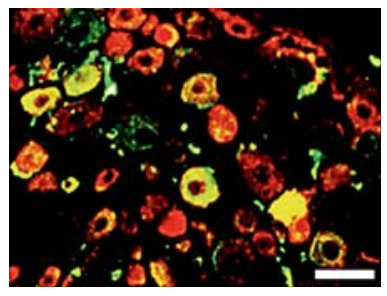

c
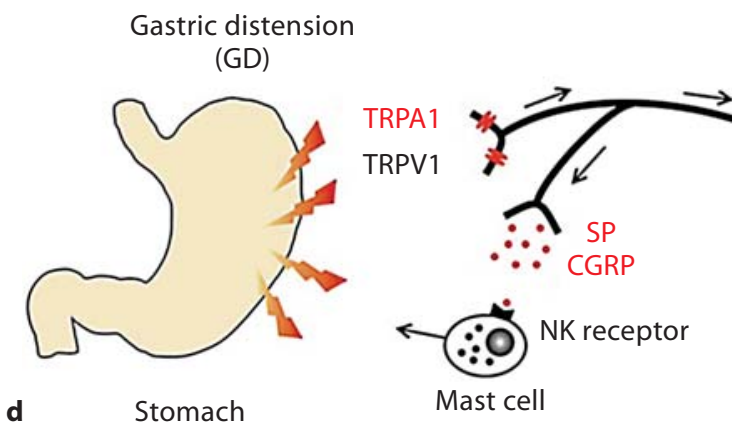

$\mathrm{p}-\mathrm{ERK} 1 / 2 \uparrow$
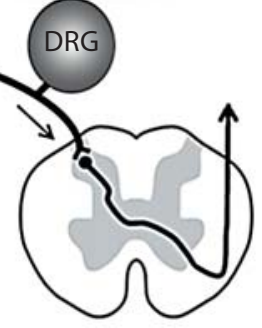

Spinal cord 


\section{Effect of Afferent Denervation on VMR and ERK1/2 Activation in DRG and NG Neurons Caused by Noxious GD}

The stomach is innervated by the vagus nerves that project via the NG in the rat (inferior vagal ganglion in humans) to the nucleus of the solitary tract in the medulla, and by spinal afferents (in splanchnic nerves), that project via the DRG to thoracic spinal cord segments [2]. It has been reported that splanchnectomy, but not vagotomy, reduces responses to gastric mechanical stimulation [13]. In contrast, vagotomy, but not splanchnectomy, abolishes VMR to intragastric acid $(\mathrm{HCl})$ administration $[6,7,13]$. Vagal and spinal neurons both contribute to mediation of responses when acid induced injury and mechanical stimuli are combined [14].

Consistent with these previous reports, we found that splanchnic nerve resection significantly affected the response to 60 and $100 \mathrm{~mm} \mathrm{Hg}$ of noxious GD, whereas subdiaphragmatic vagotomy had no effect on VMR to $100 \mathrm{~mm} \mathrm{Hg}$ of noxious GD $[8,15]$. As expected, splanchnic division significantly attenuated the noxious stimulation-induced activation of ERK1/2 in DRG but not in NG neurons [8]. These data suggest that noxious gastric mechanical stimulation (at least in the absence of preceding acid chemical injury) is primarily carried through spinal splanchnic rather than vagal afferents.

\section{Contribution of TRPA1 in Primary Afferents to Acute} Visceral Pain

Multiple mammalian transient receptor potential (TRP) genes have been cloned and classified into six subfamilies. TRP channels are highly conserved and widely expressed, contributing to the detection diverse sensory (including noxious) stimuli [16]. Experimental evidence implicates three channels: TRPV1, TRPV4 and acid-sensing ion channel 3 (ASIC3) in visceral mechanosensation $[17,18]$. The more recently identified TRP channel A1 (TRPA1) is also expressed in small sensory neurons and can be activated by a variety of stimuli, including icilin, pungent chemicals, several environmental irritants and noxious cold [19-21]. TRPA1 knockout mice have been recently reported to display impaired responses to noxious mechanical stimulation in the colon [22]. Furthermore, TRPA1 deletion significantly reduced colitis-induced mechanical hyperalgesia in the colon [23]. However, little is known about the contribution to acute visceral pain of TRPA1 in primary afferents innervating the stomach.

Role of Transient Receptor Potential A1 in Gastric Nociception
Expression of TRPA1 in Visceral Afferent Pathways

The expression of TRPA1 in thoracolumbar (T10-L1) and lumbosacral (L6-S1) DRG as well as NG has been recently reported using quantitative RT-PCR analysis and fluorescence in situ hybridization [22], and by immunohistochemistry in retrograde labeled visceral sensory neurons from stomach and distal colon [22, 23]. Moreover, TRPA1-immunoreactive (TRPA1-IR) fibers in the mouse colon were located in both mucosal and serosal/ mesenteric afferents, colocalizing with the established sensory neuropeptide, calcitonin gene-related peptide (CGRP) [22]. Consistent with these previous studies, we recently found that TRPA1-IR neurons expressed in rat DRG and NG neurons could be double labeled with fluorogold from nerves innervating the stomach. TRPA1-IR nerve fibers in the rat stomach were expressed in the mucosa, around blood vessels in the submucosa, and in the external muscle layer [15]. These data suggest that TRPA1 is expressed in vagal and spinal primary afferents innervating the rat stomach.

\section{Knockdown or Inhibition of TRPA1 Reduces VMR} and ERK1/2 Activation in Rat DRG Neurons in Response to GD

To test whether acute visceral pain after noxious mechanical stimulation of the stomach is dependent on the presence of TRPA1 in sensory neurons, an antisense oligodeoxynucleotide (AS-ODN) targeting TRPA1 was administered intrathecally. Intrathecal administration of TRPA1 AS-ODN attenuated the VMR produced by 60 and $100 \mathrm{~mm} \mathrm{Hg}$ of GD, and also suppressed ERK1/2 activation in DRG, but not NG, neurons following noxious GD [15] (fig. 2a, b). These data indicate that the TRPA1 channel is implicated in visceral mechanosensation and that the TRPA1 channel in spinal visceral afferents is required for ERK1/2 activation after noxious mechanical stimulation. Further, the majority of p-ERK1/2-labeled neurons 2 min after 60 and $100 \mathrm{~mm} \mathrm{Hg}$ of GD also coexpressed TRPA1 in DRG neurons [15] (fig. 2c).

To further confirm the functional roles of TRPA1 in primary afferent nerves innervating rat stomach, we investigated whether inhibition of TRPA1 activation modifies the response to noxious mechanical stimulation. We found that intrathecal and intraperitoneal administration of a newly identified potent and selective TRPA1 inhibitor HC-030031 completely attenuated the response to noxious GD [15]. Although the effects of HC-030031 on acute visceral pain may be mediated by the inhibition of TRPA1 activation in the spinal cord or DRG, these data at least support the hypothesis that noxious mechanical

Digestion 2010;82:150-155 
stimuli are mainly mediated via the spinal rather than vagal afferent pathway. Furthermore, these data indicate that blockade of TRPA 1 in primary afferent or spinal cord could reduce signaling of specific mechanical stimuli from the stomach and thus become a key therapeutic target for the reduction of visceral pain.

\section{Colocalization of TRPA1 with Other Nocisensors in Gastric Afferent Pathways}

The above data suggest that in response to noxious GD, generator potentials mediated via TRPA1 may promote peripheral action potentials, which in turn result in the phosphorylation of ERK1/2 in DRG and/or spinal neurons leading to visceral pain (as measured by VMR). It has been reported that overlap exists in the contribution of several non-selective cation channels, e.g. ASIC3, TRPV1, TRPV4 as well as TRPA1 in sensing of noxious stimuli by colonic visceral afferents [22-24]. We have recently demonstrated colocalization of TRPV1 and TRPA1 in small and medium-sized neurons in rat DRG and NG derived from the stomach suggesting that overlap of expression is found in the upper and lower rodent gastrointestinal tract [15].

Neurogenic inflammation is one of several mechanisms by which TRP channel activation may contribute to acute and chronic pain states. Release of neuropeptides including substance P (SP), CGRP, and neurokinin A in response to TRP can lead to activation of innate and immune inflammatory cells with peripheral sensitization thence caused by local release of neuroactive factors such as NGF $[24,25]$. Recent data indicate that SP and CGRP, released from nociceptors, can follow TRPA1 activation to induce neurogenic inflammation and somatic pain [21, 26]. A component of the neurogenic inflammation cascade may follow activation of NK receptors (e.g. NK-1 receptor (NK-1R) on endothelial cells), which thereby induce hyperalgesia. In fact, the NK-1R antagonist SR140333 markedly attenuated neurogenic plasma protein extravasation produced by an endogenous agonist for TRPA1, 4-hydroxy-2-nonenal in peripheral tissues [21]. There is now also experimental evidence suggesting that tachykinins including SP are involved in colorectal visceral nociception via NK-1 and NK-2 receptor activation [27]. Moreover, we recently reported using an NK-1R antagonist that NK-1R was involved in the generation of dyspeptic symptoms of a chronic acid reflux esophagitis model in rats [28].

\section{Conclusions}

Activation of the TRPA 1 channel in primary afferent neurons and of ERK1/2 pathways by noxious GD contribute to mechanonociceptive mechanisms in the rat. The TRPA1 channel in spinal visceral afferents is required for ERK1/2 activation after noxious mechanical stimulation. TRPA1 may be a new potential therapeutic target for visceral pain.

\section{Disclosure Statement}

The authors declare that no financial or other conflict of interest exists in relation to the content of the article.

\section{References}

1 Tack J, Talley NJ, Camilleri M, Holtmann G, Hu P, Malagelada JR, Stanghellini V: Functional gastroduodenal disorders. Gastroenterology 2006;130:1466-1479.

2 Knowles CH, Aziz Q: Basic and clinical aspects of gastrointestinal pain. Pain 2009;141: 191-209.

-3 Grundy D, Al-Chaer ED, Aziz Q, Collins SM, Ke M, Taché Y, Wood JD: Fundamentals of neurogastroenterology: basic science. Gastroenterology 2006;130:1391-1411.

-4 Tack J, Caenepeel P, Fischler B, Piessevaux H, Janssens J: Symptoms associated with hypersensitivity to gastric distention in functional dyspepsia. Gastroenterology 2001;121:526535 .
5 Tack J, Caenepeel P, Corsetti M, Janssens J: Role of tension receptors in dyspeptic patients with hypersensitivity to gastric distention. Gastroenterology 2004;127:10581066.

6 Ozaki N, Bielefeldt K, Sengupta JN, Gebhart GF: Models of gastric hyperalgesia in the rat. Am J Physiol 2002;283:G666-G676.

7 Lamb K, Kang YM, Gebhart GF, Bielefeldt K: Gastric inflammation triggers hypersensitivity to acid in awake rats. Gastroenterology 2003;125:1410-1418.
8 Sakurai J, Obata K, Ozaki N, Tokunaga A, Kobayashi K, Yamanaka H, Dai Y, Kondo T, Miyoshi K, Sugiura Y, Matsumoto T, Miwa $\mathrm{H}$, Noguchi K: Activation of extracellular signal-regulated protein kinase in sensory neurons after noxious gastric distention and its involvement in acute visceral pain in rats. Gastroenterology 2008;134:1094-1103.

-9 Ji RR, Baba H, Brenner GJ, Woolf CJ: Nociceptive-specific activation of ERK in spinal neurons contributes to pain hypersensitivity. Nat Neurosci 1999;2:1114-1119.

10 Karim F, Wang CC, Gereau RW 4th: Metabotropic glutamate receptor subtypes 1 and 5 are activators of extracellular signal-regulated kinase signaling required for inflammatory pain in mice. J Neurosci 2001;21:3771-3779. 
11 Dai Y, Iwata K, Fukuoka T, Kondo E, Tokunaga A, Yamanaka $H$, Tachibana T, Liu Y, Noguchi K: Phosphorylation of extracellular signal-regulated kinase in primary afferent neurons by noxious stimuli and its involvement in peripheral sensitization. J Neurosci 2002;22:7737-7745.

12 Impey S, Obrietan K, Wong ST, Poser S, Yano S, Wayman G, Deloulme JC, Chan G, Storm DR: Cross-talk between ERK and PKA is required for $\mathrm{Ca}^{2+}$ stimulation of CREB-dependent transcription and ERK nuclear translocation. Neuron 1998;21:869-883.

-13 Ozaki N, Gebhart GF: Characterization of mechanosensitive splanchnic nerve afferent fibers innervating the rat stomach. Am J Physiol 2001;281:G1449-G1459.

14 Kang YM, Bielefeldt K, Gebhart GF: Sensitization of mechanosensitive gastric vagal afferent fibers in the rat by thermal and chemical stimuli and gastric ulcers. J Neurophysiol 2004;91:1981-1989.

15 Kondo T, Obata K, Miyoshi K, Sakurai J, Tanaka J, Miwa H, Noguchi K: Transient receptor potential A1 mediates gastric distention-induced visceral pain in rats. Gut 2009; 58:1342-1352.

-16 Clapham DE: TRP channels as cellular sensors. Nature 2003;426:517-524.

17 Jones RC 3rd, Xu L, Gebhart GF: The mechanosensitivity of mouse colon afferent fibers and their sensitization by inflammatory mediators require transient receptor potential vanilloid-1 and acid-sensing ion channel 3. J Neurosci 2005;25:10981-10989.
18 Brierley SM, Page AJ, Hughes PA, Adam B, Liebregts T, Cooper NJ, Holtmann G, Liedtke W, Blackshaw LA: Selective role for TRPV4 ion channels in visceral sensory pathways. Gastroenterology 2008;134:2059-2069.

19 Story GM, Peier AM, Reeve AJ, Eid SR, Mosbacher J, Hricik TR, Earley TJ, Hergarden AC, Andersson DA, Hwang SW, McIntyre P, Jegla T, Bevan S, Patapoutian A: ANKTM1, a TRP-like channel expressed in nociceptive neurons, is activated by cold temperatures. Cell 2003;112:819-829.

20 Jordt SE, Bautista DM, Chuang HH, McKemy DD, Zygmunt PM, Högestätt ED, Meng ID, Julius D: Mustard oils and cannabinoids excite sensory nerve fibres through the TRP channel ANKTM1. Nature 2004;427:260265.

21 Trevisani M, Siemens J, Materazzi S, Bautista DM, Nassini R, Campi B, Imamachi N, Andrè E, Patacchini R, Cottrell GS, Gatti R, Basbaum AI, Bunnett NW, Julius D, Geppetti P: 4-Hydroxynonenal, an endogenous aldehyde, causes pain and neurogenic inflammation through activation of the irritant receptor TRPA1. Proc Natl Acad Sci USA 2007;104:13519-13524.

22 Brierley SM, Hughes PA, Page AJ, Kwan KY, Martin CM, O'Donnell TA, Cooper NJ, Harrington AM, Adam B, Liebregts T, Holtmann G, Corey DP, Rychkov GY, Blackshaw LA: The ion channel TRPA1 is required for normal mechanosensation and is modulated by algesic stimuli. Gastroenterology 2009; 137:2084-2095.
23 Cattaruzza F, Spreadbury I, Miranda-Morales M, Grady EF, Vanner SJ, Bunnett NW: Transient receptor potential ankyrin-1 has a major role in mediating visceral pain in mice. Am J Physiol 2009; Epub ahead of print.

24 Julius D, Basbaum AI: Molecular mechanisms of nociception. Nature 2001;413:203210

25 Leon A, Buriani A, Dal Toso R, Fabris M, Romanello S, Aloe L, Levi-Montalcini R: Mast cells synthesize, store, and release nerve growth factor. Proc Natl Acad Sci USA 1994; 91:3739-3743.

26 Obata K, Katsura H, Mizushima T, Yamanaka H, Kobayashi K, Dai Y, Fukuoka T, Tokunaga $\mathrm{A}$, Tominaga $\mathrm{M}$, Noguchi K: TRPA1 induced in sensory neurons contributes to cold hyperalgesia after inflammation and nerve injury. J Clin Invest 2005;115:2393-2401.

27 Kamp EH, Beck DR, Gebhart GF: Combinations of neurokinin receptor antagonists reduce visceral hyperalgesia. J Pharmacol Exp Ther 2001;299:105-113.

28 Oshima T, Koseki J, Sakurai J, Watari J, Matsumoto T, Miwa $\mathrm{H}$ : NK-1 receptor is involved in the decreased movement in a rat chronic acid reflux esophagitis model. Neurogastroenterol Motil 2010;22:579-584, e125 